\title{
A Direct Method For Predicting The High-Cycle Fatigue Regime In SMAs: Ap- plication To Nitinol Stents
}

\author{
Pierre Colombé ${ }^{1, a}$ and Michaël Peigney $2, b$ \\ ${ }^{1}$ Département Génie Mécanique et Matériaux, Ecole des Ponts Paris, F-77455 Marne la Vallée, France \\ ${ }^{2}$ Univ Paris-Est, Laboratoire Navier (UMR 8205), CNRS, Ecole des Ponts ParisTech, IFSTTAR, F-77455 Marne la Vallée, France
}

\begin{abstract}
In fatigue design of metals, it is common practice to distinguish between high-cycle fatigue (occurring after 10000-100000 cycles) and low-cycle fatigue. For elastic-plastic materials, there is an established correlation between fatigue and energy dissipation. In particular, high-cycle fatigue occurs when the energy dissipation remains bounded in time. Although the physical mechanisms in SMAs differ from plasticity, the hysteresis observed in the stress-strain response shows that some energy dissipation occurs, and it can be reasonably assumed that situations where the energy dissipation remains bounded is the most favorable for fatigue design. We present a direct method for determining if the energy dissipation in a SMA structure is bounded or not. That method relies only on elastic calculations, thus bypassing incremental nonlinear analysis. Moreover, only a partial knowledge of the loading (namely the extreme values) is needed. Some results related to Nitinol stents are presented.
\end{abstract}

\section{Introduction}

This paper is concerned with the long-time behaviour of Shape Memory Alloys (SMA) bodies under prescribed loading histories. For elastic perfectly plastic bodies, a fundamental result is the Melan's theorem [1,2] which gives a sufficient condition for the energy dissipation to remain bounded with respect to time. That last situation is classically referred to as shakedown, and is associated with the intuitive idea that the body behaves elastically for time $t$ sufficiently large, i.e. that the plastic strain tends to a limit as $t \rightarrow \infty$. Melan's theorem has the distinctive property of being path-independent, i.e. independent on the initial state of the structure. Regarding fatigue design, shakedown corresponds to the most beneficial regime of high-cycle fatigue, as opposed to the regime of low-cycle fatigue which typically occurs if the plastic strain does not converge towards a stabilized value [3].

Much effort has been devoted to developing constitutive laws for describing the behaviour of SMAs. The phase transformation is typically described by an internal variable $\boldsymbol{\alpha}$ which - depending on the complexity of the material model - may be scalar or vectorial. A fundamental observation is that the internal variable $\alpha$ must comply with some a priori inequalities that result from the mass conservation in the phase transformation process. As a consequence, the internal variable $\alpha$ is constrained to take values in a set $\mathcal{K}$ that is not a vectorial space (in most of SMA models, $\mathcal{K}$ is a bounded set). The presence of such constraints constitutes a crucial difference with plasticity

\footnotetext{
ae-mail: colombep@eleves.enpc.f

be-mail: michael.peigney@enpc.fr
}

models, and calls for special attention when the structural evolution problem is considered [4-7].

It was recently proved that the Melan's theorem could be extended to SMAs $[8,9]$. When the shakedown limit provided by that theorem is exceeded, it was found that the large-time behaviour is dependent on the initial state: in the case of cyclic loadings, some initial conditions lead to shakedown whereas some others lead to alternating phase transformation. Such a feature is not found in standard plasticity. The shakedown theorem in $[8,9]$ is pathindependent - in the spirit of the original Melan theorem $[1,2]$ - and applies to a wide range of constitutive models of phase transformation in SMAs. As explained in this paper, the theorem in $[8,9]$ can be used to obtain bounds on the loadings for which shakedown occurs, thus leading to a general method for the fatigue design of SMA structures.

\section{Evolution of a SMA continuum}

\subsection{Constitutive laws}

We consider constitutive SMA models which enter the framework of standard generalized materials [10]. The local state of the material is described by the strain $\epsilon$ and an internal variable $\alpha$ tracking the phase transformation. The variable $\alpha$ is constrained to take values in a given bounded set $\mathcal{K}$. The rate-independent behavior of the material is determined by the constitutive relations

$$
\begin{gathered}
\sigma=\frac{\partial w}{\partial \boldsymbol{\epsilon}}(\boldsymbol{\epsilon}, \boldsymbol{\alpha}), \boldsymbol{A}=-\frac{\partial w}{\partial \boldsymbol{\alpha}}(\boldsymbol{\epsilon}, \boldsymbol{\alpha}), \\
\boldsymbol{A}=\boldsymbol{A}^{d}+\boldsymbol{A}^{r},
\end{gathered}
$$




$$
\begin{aligned}
& \dot{\boldsymbol{\alpha}} \in \partial I_{C}\left(\boldsymbol{A}^{d}\right), \\
& \boldsymbol{A}^{r} \in \partial I_{\mathcal{K}}(\boldsymbol{\alpha}) .
\end{aligned}
$$

where $w(\boldsymbol{\epsilon}, \boldsymbol{\alpha})$ is the free energy function and $C$ is the elasticity domain of the material. In (3)-(4), $\partial$ denotes the subdifferential operator [11] and $I_{\mathcal{K}}\left(\right.$ resp. $\left.I_{C}\right)$ is the indicator function of the set $\mathcal{K}$ (resp. $\mathcal{C}$ ), i.e. the function that is equal to 0 in $\mathcal{K}$ (resp. $C$ ) and infinite outside $\mathcal{K}$ (resp. $C$ ). In (2), $\boldsymbol{A}$ can be interpreted as the driving force for phase transformation. Eq. (3) is the normality flow rule. The term $\boldsymbol{A}^{r}$ in (2-4) arises as a consequence of the constraint $\alpha \in \mathcal{K}$. We refer to [12] for a derivation of (1-4) from the principle of thermodynamics.

We assume that the sets $C, \mathcal{K}$ are convex and consider free energy functions $w(\boldsymbol{\epsilon}, \boldsymbol{\alpha})$ of the form

$$
w(\boldsymbol{\epsilon}, \boldsymbol{\alpha})=\frac{1}{2}(\boldsymbol{\epsilon}-\boldsymbol{K} \cdot \boldsymbol{\alpha}): \boldsymbol{L}:(\boldsymbol{\epsilon}-\boldsymbol{K} \cdot \boldsymbol{\alpha})+f(\boldsymbol{\alpha})
$$

where $\boldsymbol{L}$ is symmetric positive, $\boldsymbol{K}$ is a given matrix, and $f$ is a positive differentiable function (not necessarily linear nor convex). In such case, we have from (1)

$$
\boldsymbol{\sigma}=\boldsymbol{L}:(\boldsymbol{\epsilon}-\boldsymbol{K} \cdot \boldsymbol{\alpha}), \boldsymbol{A}=\boldsymbol{K}^{t}: \boldsymbol{\sigma}-f^{\prime}(\boldsymbol{\alpha})
$$

where $\boldsymbol{K}^{t}$ is the transpose of $\boldsymbol{K}$ and $f^{\prime}$ is the derivative of $f$. The total strain $\boldsymbol{\epsilon}$ is thus the sum of an elastic strain $\boldsymbol{L}^{-1}: \boldsymbol{\sigma}$ (proportional to the stress) and an inelastic strain $\boldsymbol{K} \cdot \boldsymbol{\alpha}$ (proportional to the internal variable $\boldsymbol{\alpha}$ ). The driving force $\boldsymbol{A}$ depends linearly on the stress $\boldsymbol{\sigma}$, but may depend nonlinearly on the internal variable $\alpha$.

Many existing models of shape-memory alloys fit in the format considered. For instance, a three-dimensional micromechanical model of single crystals used in the literature $[4,6,7]$ is given by

$$
\begin{aligned}
& w(\boldsymbol{\epsilon}, \boldsymbol{\xi})=\frac{1}{2}\left(\boldsymbol{\epsilon}-\sum_{i=1}^{n} \xi_{i} \boldsymbol{\epsilon}_{i}\right): \boldsymbol{L}:\left(\boldsymbol{\epsilon}-\sum_{i=1}^{n} \xi_{i} \boldsymbol{\epsilon}_{i}\right)+\sum_{i=1} m_{i} \xi_{i}, \\
& \mathcal{C}=\left[G_{1}^{-}, G_{1}^{+}\right] \times \cdots \times\left[G_{n}^{-}, G_{n}^{+}\right], \\
& \mathcal{K}=\left\{\boldsymbol{\xi} \in \mathbb{R}_{+}^{n}: \sum_{i=1}^{n} \xi_{i} \leq 1\right\},
\end{aligned}
$$

where $n$ is the number of martensitic variants and $\boldsymbol{\epsilon}_{i}$ is the given transformation strain for each variant. The scalars $m_{i}, G_{i}^{+}, G_{i}^{-}$in (6) are all constitutive parameters of the model. The internal variable $\boldsymbol{\xi}=\left(\xi_{1}, \cdots, \xi_{n}\right)$ represents the set of volume fractions for each martensitic variant. Because of mass conservation in the phase transformation, the volume fraction of the austenite is equal to $1-\sum_{i} \xi_{i}$, hence the constraint $\sum_{i} \xi_{i} \leq 1$ that is imposed on $\boldsymbol{\xi}$. For $\boldsymbol{\xi}$ verifying $0<\xi_{i}$ (for all $i$ ) and $\sum_{i} \xi_{i}<1$, the term $\boldsymbol{A}^{r}$ in (4) is equal to 0 and the relations (2-3) become

$$
\begin{array}{ll}
\dot{\xi}_{i} \geq 0 & \text { if } \boldsymbol{\sigma}: \boldsymbol{\epsilon}_{i}-m_{i}=G_{i}^{+}, \\
\dot{\xi}_{i} \leq 0 & \text { if } \boldsymbol{\sigma}: \boldsymbol{\epsilon}_{i}-m_{i}=G_{i}^{-}, \\
\dot{\xi}_{i}=0 & \text { if } \boldsymbol{\sigma}: \boldsymbol{\epsilon}_{i}-m_{i} \in\left(G_{i}^{-}, G_{i}^{+}\right)
\end{array}
$$

Those relations define the evolution of phasetransformation in a way similar to crystal plasticity.
Phenomenological SMA models can also enter the format considered. An example is the model of [13, 14], defined by

$$
\begin{aligned}
& w\left(\boldsymbol{\epsilon}, \boldsymbol{\alpha}^{t r}\right)=\frac{1}{2}\left(\boldsymbol{\epsilon}-\boldsymbol{\alpha}^{t r}\right): \boldsymbol{L}:\left(\boldsymbol{\epsilon}-\boldsymbol{\alpha}^{t r}\right)+a\left\|\boldsymbol{\alpha}^{t r}\right\|+\frac{1}{2} h\left\|\boldsymbol{\alpha}^{t r}\right\|^{2}, \\
& \mathcal{C}=\left\{\sigma \in \mathbb{R}_{s}^{3 \times 3}:\|\operatorname{dev}(\boldsymbol{\sigma})\| \leq \sigma_{Y}\right\} \\
& \mathcal{K}=\left\{\boldsymbol{\alpha}^{t r} \in \mathbb{R}_{S}^{3 \times 3}: \operatorname{tr} \boldsymbol{\alpha}^{t r}=0 ;\left\|\boldsymbol{\alpha}^{t r}\right\| \leq \epsilon_{L}\right\} .
\end{aligned}
$$

where dev denotes the deviator. In the model (7), the internal variable is the macroscopic transformation strain $\boldsymbol{\alpha}^{t r}$. The constitutive parameters $h, \epsilon_{L}$ and $\sigma_{Y}$ are positive. The constitutive parameter $a$ is positive in the superelastic regime, i.e. for sufficiently high temperatures. The norm $\|$.$\| in (7) is the euclidean norm in the space of 3 \times 3$ symmetric matrices, i.e. $\|\tau\|=\sqrt{\sum_{i, j=1}^{3} \tau_{i j}^{2}}$. In order to elucidate the meaning of the relations (2-4), consider the simplifying case $a=h=0$ in (7). For $\boldsymbol{\alpha}^{t r}$ such that $\left\|\boldsymbol{\alpha}^{t r}\right\|<\epsilon_{L}$, the term $\boldsymbol{A}^{r}$ in (4) is equal to 0 so that (2-3) reduce to

$$
\dot{\boldsymbol{\alpha}}^{t r}=\lambda \operatorname{dev}(\sigma) \text { with } \lambda \geq 0, \lambda\left(\sigma_{Y}-\|\operatorname{dev}(\sigma)\|\right)=0 .
$$

The relation (8) corresponds the Von Mises flow rule for the internal variable $\boldsymbol{\alpha}^{t r}$. In particular, when $\dot{\boldsymbol{\alpha}}^{t r} \neq 0$ (i.e. phase transformation occurs), (8) can be equivalently rewritten as

$$
\operatorname{dev}(\sigma)=\sigma_{Y} \frac{\dot{\boldsymbol{\alpha}}^{t r}}{\left\|\dot{\boldsymbol{\alpha}}^{t r}\right\|} .
$$

For $\boldsymbol{\alpha}^{t r}$ such that $\left\|\boldsymbol{\alpha}^{t r}\right\|=\epsilon_{L}$, the term $\boldsymbol{A}^{r}$ in (4) takes the form $\boldsymbol{A}^{r}=\mu \boldsymbol{\alpha}$ (with $\mu \geq 0$ ). Eqs (8-9) have to be modified accordingly. In particular, (9) becomes

$$
\operatorname{dev}(\sigma)=\sigma_{Y} \frac{\dot{\boldsymbol{\alpha}}^{t r}}{\left\|\dot{\boldsymbol{\alpha}}^{t r}\right\|}+\mu \boldsymbol{\alpha}
$$

\subsection{Quasi-static evolutions}

In the following we are interested in studying the evolutions of a continuum submitted to a prescribed loading history. The continuum occupies a domain $\Omega$ and is submitted to body forces $\boldsymbol{f}^{d}$. Displacements $\boldsymbol{u}^{d}$ are imposed on a part $\Gamma_{u}$ of the boundary $\Gamma$, and tractions $\boldsymbol{T}^{d}$ are prescribed on $\Gamma_{T}=\Gamma-\Gamma_{u}$. The given functions $\boldsymbol{f}^{d}, \boldsymbol{u}^{d}, \boldsymbol{T}^{d}$ depend on position $\boldsymbol{x}$ and time $t$. The stress and state variables $(\boldsymbol{\sigma}, \boldsymbol{\epsilon}, \boldsymbol{\alpha})$ in the continuum are also expected to depend on $(\boldsymbol{x}, t)$. In order to alleviate the expressions, this dependence will be omitted in the notations, unless in the case of possible ambiguities.

Quasi-static evolutions of the continuum are governed by the following system:

$$
\begin{gathered}
\boldsymbol{\sigma} \in \mathcal{K}_{\sigma}, \boldsymbol{\epsilon} \in \mathcal{K}_{\epsilon}, \boldsymbol{\alpha} \in \mathcal{K}, \\
\dot{\boldsymbol{\alpha}} \in \partial I_{C}\left(\boldsymbol{A}^{d}\right), \boldsymbol{A}^{r} \in \partial I_{\mathcal{K}}(\boldsymbol{\alpha}), \\
\boldsymbol{\sigma}=\boldsymbol{L}:(\boldsymbol{\epsilon}-\boldsymbol{K} \cdot \boldsymbol{\alpha}), \\
\boldsymbol{K}^{t}: \boldsymbol{\sigma}-f^{\prime}(\boldsymbol{\alpha})=\boldsymbol{A}^{d}+\boldsymbol{A}^{r},
\end{gathered}
$$

where $\mathcal{K}_{\sigma}$ and $\mathcal{K}_{\epsilon}$ are respectively the sets of admissible stress and strain fields, defined by

$$
\begin{aligned}
& \mathcal{K}_{\sigma}=\left\{\sigma \mid \operatorname{div} \boldsymbol{\sigma}+\boldsymbol{f}^{d}=0 \text { in } \Omega ; \boldsymbol{\sigma} \cdot \boldsymbol{n}=\boldsymbol{T}^{d} \text { on } \Gamma_{T}\right\}, \\
& \mathcal{K}_{\epsilon}=\left\{\boldsymbol{\epsilon} \mid \boldsymbol{\epsilon}=\left(\nabla \boldsymbol{u}+(\nabla \boldsymbol{u})^{t}\right) / 2 \text { in } \Omega ; \boldsymbol{u}=\boldsymbol{u}^{d} \text { on } \Gamma_{u}\right\} .
\end{aligned}
$$

Starting from a given initial state $\alpha(0),(10)$ determines the evolution of the stress and strain fields. 


\section{A general method for fatigue design}

\subsection{Shakedown theorem}

We examine conditions under which the energy dissipation $\int_{0}^{T} \int_{\Omega} \boldsymbol{A}^{d} \cdot \dot{\boldsymbol{\alpha}} d \boldsymbol{x} d t$ remains bounded (with respect to time $T$ ) for all solutions of the evolution problem (10). Such a situation is referred to as shakedown. As mentioned in the introduction, shakedown is related to the fact that the evolution becomes elastic in the large-time limit $[8,15]$ and corresponds to high-cycle fatigue.

Let us introduce the so-called fictitious elastic response $\left(\sigma^{E}, \epsilon^{E}\right)$ of the system, i.e. the response that would be obtained if the material was purely elastic. More precisely, $\left(\sigma^{E}, \epsilon^{E}\right)$ is the solution of

$$
\boldsymbol{\sigma}^{E} \in \mathcal{K}_{\sigma}, \boldsymbol{\epsilon}^{E} \in \mathcal{K}_{\epsilon}, \sigma^{E}=\boldsymbol{L}: \boldsymbol{\epsilon}^{E}
$$

The central result is the following shakedown theorem [8, 9]: If there exists $m>1, \tau \geq 0$ and a time-independent field $\boldsymbol{A}^{*}(\boldsymbol{x})$ such that

$$
m \boldsymbol{K}^{t}: \boldsymbol{\sigma}^{E}(\boldsymbol{x}, t)-\boldsymbol{A}^{*}(\boldsymbol{x}) \in C \forall \boldsymbol{x} \in \Omega, \forall t>\tau
$$

then there is shakedown, irrespective of the initial condition. A proof of that theorem is presented in 3.2. We refer to $[8,9]$ for more details.

There is a simple geometric interpretation of the condition (13): Consider a fixed location $\boldsymbol{x}$ and let $\Gamma(t)$ be the curve described by $\boldsymbol{K}^{t}: \boldsymbol{\sigma}^{E}(\boldsymbol{x}, t)$. The condition (13) means that, up to a time-independent translation, the curve $\Gamma$ remains in the elasticity domain $C$ (for time $t$ large enough).

As an example, consider the material model (7). Using the presented theorem, it can easily be seen that shakedown occurs if $\left\|m \boldsymbol{s}^{E}(\boldsymbol{x}, t)-\boldsymbol{A}^{*}(\boldsymbol{x})\right\| \leq \sigma_{Y}$ where $\boldsymbol{s}^{E}$ is the deviatoric part of $\boldsymbol{\sigma}^{E}$ and $\boldsymbol{A}^{*}(\boldsymbol{x})$ is an arbitrary deviatoric tensor. The obtained shakedown condition thus reduces to a restriction on the diameter of the curve $s^{E}(x, t)$, as for shakedown in linear kinematic hardening plasticity $[15,16]$.

Observe that we did not assume the convexity of $f$. This is a welcome feature for the shakedown analysis of SMA bodies because the function $f$ associated with some micromechanical SMA models is not necessarily convex [17-20]. We refer to [9] for a detailed discussion between the theorem (13) and the analog result in plasticity.

Finally, we note that the above theorem gives a sufficient condition for shakedown to occur, whatever the initial state is. When the loading is beyond the limit provided by the theorem, shakedown may still occur for some (but not all) initial conditions (see [8,9]) for some examples). In such case, the asymptotic behavior is strongly dependent on the initial state: For instance, in the case of periodic loading, some initial conditions leads to shakedown while others lead to alternate phase transformation (i.e. a periodic but non constant evolution of the phase transformation). Interesting, such dependence of the asymptotic regime on the initial state has also been observed in other nonlinear mechanical problems, such as contact with friction [21] and plasticity with temperature-dependent elastic moduli [22].

\subsection{Proof of the theorem}

Consider a solution $\left(\boldsymbol{\epsilon}, \boldsymbol{\alpha}, \boldsymbol{\sigma}, \boldsymbol{A}^{r}, \boldsymbol{A}^{d}\right)$ of the evolution problem (for some given initial condition) and let

$$
D(t)=\int_{\Omega} \boldsymbol{A}^{d} \cdot \dot{\boldsymbol{\alpha}} d \boldsymbol{x}
$$

be the rate of dissipated energy. Assuming $\left(m, \tau, \boldsymbol{A}_{*}^{r}\right)$ satisfy (13), we show in the following that the total dissipated energy $\int_{0}^{T} D(t) d t$ remains bounded as $T \rightarrow \infty$. To that purpose, introduce the positive functional $W(t)$ defined as

$$
W(t)=\int_{\Omega} w\left(\boldsymbol{\epsilon}(t)-\boldsymbol{\epsilon}^{E}(t), \boldsymbol{\alpha}(t)\right) d \boldsymbol{x} .
$$

We have

$$
\dot{W}(t)=\int_{\Omega}\left[\left(\boldsymbol{\sigma}-\boldsymbol{\sigma}^{E}\right):\left(\dot{\boldsymbol{\epsilon}}-\dot{\boldsymbol{\epsilon}}^{E}-\boldsymbol{K} \cdot \dot{\boldsymbol{\alpha}}\right)+f^{\prime}(\boldsymbol{\alpha}) \cdot \dot{\boldsymbol{\alpha}}\right] d \boldsymbol{x} .
$$

Since $\operatorname{div}\left(\boldsymbol{\sigma}-\boldsymbol{\sigma}^{E}\right)=0$ in $\Omega,\left(\boldsymbol{\sigma}-\boldsymbol{\sigma}^{E}\right) \cdot \boldsymbol{n}=0$ on $\Gamma_{T}$ and $\boldsymbol{u}-\boldsymbol{u}^{E}=0$ on $\Gamma_{u}$, the principle of virtual power gives

$$
\int_{\Omega}\left(\boldsymbol{\sigma}-\boldsymbol{\sigma}^{E}\right):\left(\dot{\boldsymbol{\epsilon}}-\dot{\boldsymbol{\epsilon}}^{E}\right) d \boldsymbol{x}=0
$$

Hence

$$
\dot{W}(t)=\int_{\Omega}\left[-\boldsymbol{K}^{t}:\left(\boldsymbol{\sigma}-\boldsymbol{\sigma}^{E}\right)+f^{\prime}(\boldsymbol{\alpha})\right] \cdot \dot{\boldsymbol{\alpha}} d \boldsymbol{x}
$$

which using (10) can be rewritten as

$$
\dot{W}(t)=-D(t)+\int_{\Omega}\left[-A^{r}+\boldsymbol{K}^{t}: \boldsymbol{\sigma}^{E}\right] \cdot \dot{\boldsymbol{\alpha}} d \boldsymbol{x} .
$$

Setting $\boldsymbol{A}_{*}^{d}=m \boldsymbol{K}^{t}: \boldsymbol{\sigma}^{E}(t)-\boldsymbol{A}_{*}^{r}$, we obtain

$$
\dot{W}(t)=-D(t)+\int_{\Omega}\left[-\boldsymbol{A}^{r}+\frac{1}{m}\left(\boldsymbol{A}_{*}^{d}+\boldsymbol{A}_{*}^{r}\right)\right] \cdot \dot{\boldsymbol{\alpha}} d \boldsymbol{x} .
$$

The property (13) shows that $\boldsymbol{A}_{*}^{d} \in C$ for $t>\tau$. Since $\dot{\alpha} \in \partial I_{C}\left(A^{d}\right)$ and $C$ is convex, we have

$$
\left(\boldsymbol{A}^{d}-\boldsymbol{A}_{*}^{d}\right) \cdot \dot{\alpha} \geq 0 .
$$

This last relation can be interpreted as the principle of maximum dissipation. Similarly, the relations $\boldsymbol{A}^{r} \in$ $\partial I_{\mathcal{K}}(\alpha)$ and $\alpha \in \mathcal{K}$ imply that

$$
0 \geq \boldsymbol{A}^{r}(t) \cdot\left(\alpha\left(t^{\prime}\right)-\alpha(t)\right)
$$

for any $t^{\prime}$. Taking the limit $t^{\prime} \longrightarrow t$ with $t^{\prime}<t$, we obtain

$$
\boldsymbol{A}^{r} \cdot \dot{\boldsymbol{\alpha}} \geq 0
$$

where $\dot{\alpha}$ is the left-time derivative. Combining (15)-(16) with (14) yields

$$
\dot{W}(t) \leq \frac{1-m}{m} D(t)+\frac{1}{m} \int_{\Omega} \boldsymbol{A}_{*}^{r} \cdot \dot{\boldsymbol{\alpha}} d \boldsymbol{x} .
$$

Integrating with respect to time on $[\tau, T]$ and noting that $\boldsymbol{A}_{*}^{r}$ is time-independent, we find

$$
(m-1) \int_{\tau}^{T} D(t) d t \leq m W(\tau)+\int_{\Omega} \boldsymbol{A}_{*}^{r} \cdot(\boldsymbol{\alpha}(T)-\boldsymbol{\alpha}(\tau)) d \boldsymbol{x}
$$


where the property $W(T) \geq 0$ has been used. Since $\mathcal{K}$ is bounded, there exists a positive constant $K$ such that $\|\boldsymbol{\alpha}\| \leq K$ for any $\boldsymbol{\alpha} \in \mathcal{K}$. Therefore

$$
\int_{\Omega} \boldsymbol{A}_{*}^{r} \cdot(\boldsymbol{\alpha}(t)-\boldsymbol{\alpha}(\tau)) d \boldsymbol{x} \leq 2 K \int_{\Omega}\left\|\boldsymbol{A}_{*}^{r}\right\| d \boldsymbol{x} .
$$

Combining that inequality with (17) gives

$$
(m-1) \int_{\tau}^{T} D(t) d t \leq m W(\tau)+2 K \int_{\Omega}\left\|\boldsymbol{A}_{*}^{r}\right\| d \boldsymbol{x} .
$$

The right-hand side of that inequality is independent on $T$. Therefore $\int_{\tau}^{T} D(t)$ is bounded as $T \longrightarrow+\infty$, which completes the proof.

\subsection{Application to fatigue design}

In practice, the shakedown condition (13) can be used to bound the loadings for which shakedown occurs. To illustrate this point, consider the simple situation of a proportional loading history: The functions $\boldsymbol{f}^{d}, \boldsymbol{u}^{d}, \boldsymbol{T}^{d}$ that define the loading history take the form

$$
\begin{gathered}
\boldsymbol{f}^{d}(\boldsymbol{x}, t)=\lambda(t) \boldsymbol{f}_{0}^{d}(\boldsymbol{x}), \\
\boldsymbol{u}^{d}(\boldsymbol{x}, t)=\lambda(t) \boldsymbol{u}_{0}^{d}(\boldsymbol{x}) \\
\boldsymbol{T}^{d}(\boldsymbol{x}, t)=\lambda(t) \boldsymbol{T}_{0}^{d}(\boldsymbol{x})
\end{gathered}
$$

where $\lambda(t)$ is a loading parameter, depending on time. The elastic response $\sigma^{E}$ in (12) can be written as

$$
\sigma^{E}(x, t)=\lambda(t) \sigma_{0}^{E}(x)
$$

where $\sigma_{0}^{E}(\boldsymbol{x})$ is the solution of the elasticity problem (12) for the loading $\left(\boldsymbol{f}_{0}^{d}, \boldsymbol{u}_{0}^{d}, \boldsymbol{T}_{0}^{d}\right)$. Therefore, the condition (13) becomes

$$
m \lambda(t) \boldsymbol{K}^{t}: \boldsymbol{\sigma}_{0}^{E}(\boldsymbol{x})-\boldsymbol{A}^{*}(\boldsymbol{x}) \in \mathcal{C} \forall \boldsymbol{x} \in \Omega, \forall t>\tau
$$

Assume that $\lambda(t)$ varies between a minimum value $\lambda_{\text {min }}$ and a maximum value $\lambda_{\max }$. In such case, (18) is satisfied provided that

$$
m\left(\lambda_{\max }-\lambda_{\min }\right) \boldsymbol{K}^{t}: \boldsymbol{\sigma}_{0}^{E}(\boldsymbol{x})-\boldsymbol{A}^{*}(\boldsymbol{x}) \in C
$$

for some $\boldsymbol{A}^{*}(\boldsymbol{x})$ and some $m>1$. It is necessary at this point to distinguish between the SMA models considered. For instance, for the micromechanical model (6) of single crystals, the condition (19) can be rewritten as

$$
m\left(\lambda_{\max }-\lambda_{\min }\right) \boldsymbol{\epsilon}^{i}: \boldsymbol{\sigma}_{0}^{E}(\boldsymbol{x}) \leq G_{i}^{+}-G_{i}^{-}
$$

where we recall that $\boldsymbol{\epsilon}^{i}$ is the transformation strain for variant $i$. For a scalar $m>1$ satisfying (20) at all point $\boldsymbol{x}$ to exist, it is necessary that

$$
\left(\lambda_{\max }-\lambda_{\min }\right)\left\{\sup _{\boldsymbol{x} \in \Omega} \boldsymbol{\epsilon}^{i}: \boldsymbol{\sigma}_{0}^{E}(\boldsymbol{x})\right\}<G_{i}^{+}-G_{i}^{-} .
$$

The conclusion is that shakedown occurs if the loading satisfies

$$
\lambda_{\max }-\lambda_{\min }<M_{H C F}
$$

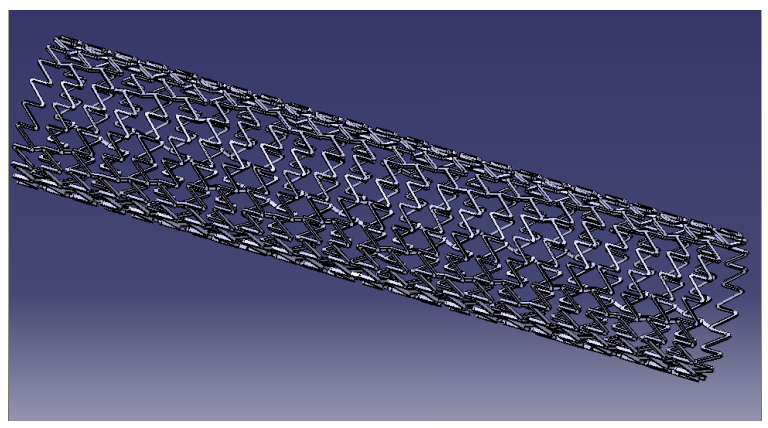

Figure 1. Geometry of a stent.

where

$$
M_{H C F}=\min _{i} \frac{G_{i}^{+}-G_{i}^{-}}{\sup _{\boldsymbol{x} \in \Omega} \boldsymbol{\epsilon}^{i}: \sigma_{0}^{E}(\boldsymbol{x})} .
$$

We can observe that the loading history only appears in (21) through the extreme values $\lambda_{\min }$ and $\lambda_{\max }$ : The detailed knowledge of $\lambda(t)$ is not required. In particular, $\lambda(t)$ does not need to be periodic.

As an other example, consider the case where the elasticity domain $C$ is a ball, such as for the phenomenological model (7). Then (19) can be satisfied by some $\boldsymbol{A}^{*}(\boldsymbol{x})$ provided that

$$
m\left(\lambda_{\max }-\lambda_{\min }\right)\left\|\boldsymbol{K}^{t}: \boldsymbol{\sigma}_{0}^{E}(\boldsymbol{x})\right\| \leq \operatorname{diam} C \forall \boldsymbol{x} \in \Omega
$$

where $\operatorname{diam} C$ is the diameter of $C$, i.e.

$$
\operatorname{diam} C=\sup _{\tau, \tau^{\prime} \in C}\left\|\tau-\tau^{\prime}\right\|
$$

Shakedown occurs if (23) is satisfied by some $m>1$ and for all $x \in \Omega$, i.e. if

$$
\lambda_{\max }-\lambda_{\min }<M_{H C F}
$$

where $M_{H C F}$ is now defined by

$$
M_{H C F}=\frac{\operatorname{diam} C}{\sup _{\boldsymbol{x} \in \Omega}\left\|\boldsymbol{K}^{t}: \boldsymbol{\sigma}_{0}^{E}(\boldsymbol{x})\right\|} .
$$

The conclusion is the following: If the amplitude $\lambda_{\text {max }}-\lambda_{\text {min }}$ of the loading is smaller than $M_{H C F}$, then the structure shakes down and therefore is expected to be in the high cycle fatigue regime. The scalar $M_{H C F}$ can thus be interpreted as a limit for high cycle fatigue. In practice, the calculation of $M_{H C F}$ only requires to solve a single problem of linear elasticity (so as to obtain $\sigma_{0}^{E}$ ). The relation (21) can be used as a criterion for the fatigue design of SMA structures: If the amplitude of the loading is specified, the geometry of the structures and the choice of constitutive materials should be adjusted so as to satisfy (24). The latter indeed have a direct influence of the elastic response $\sigma_{0}^{E}$.

\section{Application to Nitinol stents}

In this section we discuss the application of the proposed method to Nitinol stents, that are used in the biomedical 

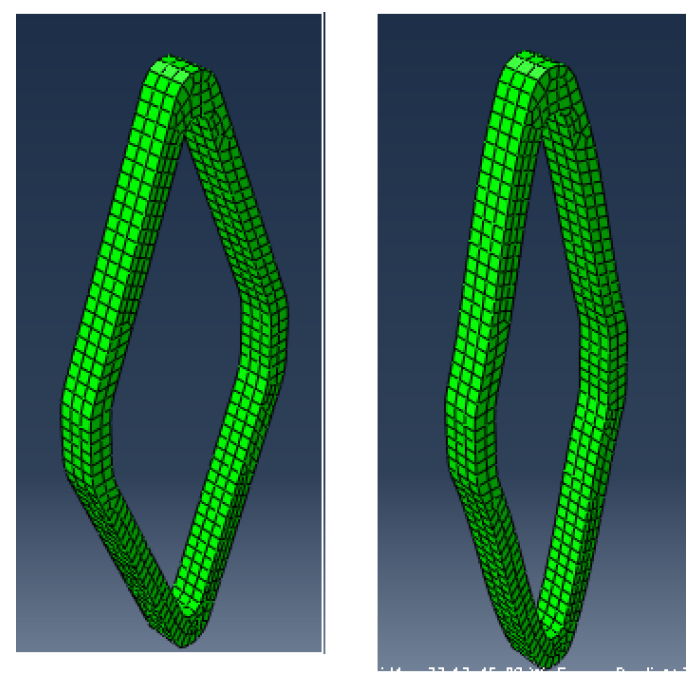

Figure 2. Diamond-shape specimen used in unaxial traction tests: Reference configuration (left), Deformed configuration (right).

field for treating artery disease [23, 24]. The tubular geometry of such devices (see Fig. 1) usually consists in the repetition of an elementary motif (a 'strut $\mathrm{V}$ ' in the case of Fig. 1). In order to study the fatigue of stents, some extensive testing has been done in [25] on specifically designed diamond-shape specimen (a simplified model of such specimen is shown in Fig. 2). Those diamond shaped specimen consists in 2 'strut Vs' arranged in a symmetric fashion, so as to be easily fitted in a fatigue test machine. The specimen were submitted to displacement-controlled cyclic loading (along the horizontal direction in Fig. 2), with prescribed mean and strain amplitudes. The resulting S-N curves (strain amplitude vs number of cycles to failure) showed a low- to high-cycle fatigue transition occurring for a strain amplitude approximatively equal to $0.5 \%$, without any clear influence of the mean strain: for a prescribed strain amplitude larger than $0.5 \%$, failure typically occurred after a low number of cycles $\left(10^{3}-10^{5}\right)$, whereas for a prescribed strain amplitude smaller than $0.5 \%$, the specimen survived after $10^{5}$ cycles (see [25] for details).

It is interesting to see if the shakedown-based approach presented in Sect. 3 is consistent with then experimental results in [25]. To that purpose, linear Finite Element Analysis (FEA) was used to evaluate the fictitious elastic response $\sigma_{0}^{E}$. The geometry used in the computations is represented in Fig. 2(left). The maximum length along the horizontal direction (which is the direction of loading) is $l=1.35 \mathrm{~mm}$. The length in the vertical direction is equal to $3.104 \mathrm{~mm}$ and the thickness is equal to $0.2 \mathrm{~mm}$. In Fig. 2(right) is shown the deformed configuration obtained by FEA for a reference value $\epsilon^{0}$ of the imposed strain (arbitrary set equal to $0.055 / l=0.0407 \%$ ). The corresponding stress distribution $\sigma_{0}^{E}$ is shown in Fig. 3. The elasticity tensor $\boldsymbol{L}$ was chosen as isotropic with a Young modulus equal to $50 \mathrm{GPa}$ and a Poisson ration equal to 0.35 [14].

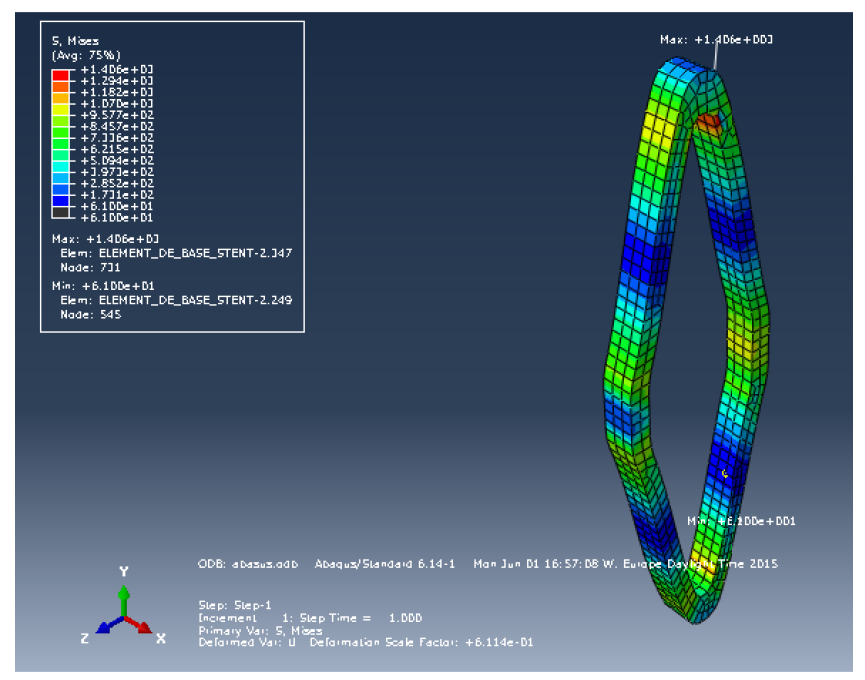

Figure 3. Results of linear FEA (Von Mises distribution).

Using the SMA model (7), the high cycle fatigue limit (24) becomes

$$
M_{H C F}=\frac{2 \sigma_{Y}}{\sup _{\boldsymbol{x} \in \Omega}\left\|\boldsymbol{s}_{0}^{E}(\boldsymbol{x})\right\|} .
$$

Note that $\left\|\boldsymbol{s}_{0}^{E}(\boldsymbol{x})\right\|$ corresponds to the standard Von Mises stress. FEA shows that $\sup \left\|s_{0}^{E}(\boldsymbol{x})\right\|$ is approximatively equal to $1406 \mathrm{MPa}$. That value is reached in the knee area of the specimen (see Fig. 3 ). Using the constitutive value $\sigma_{Y}=50 \mathrm{MPa}$ [14], the shakedown limit is approximatively equal to $0.35 \%$ (strain amplitude), which -notably given the approximation on the geometry as well as on the constitutive parameters - is in satisfactory agreement with the experimental results of [25].

\section{Concluding remarks}

In this communication, we have presented a general method for the fatigue design of SMA bodies, based on shakedown theory. That method is direct in the sense that incremental nonlinear analysis - which is costly and somewhat tricky to perform for SMAs - is completely bypassed. Moreover, only a partial knowledge of the loading (namely the knowledge of the extreme values) is required. That method is very simple to use as it only relies on elastic calculations and can be implemented in the postprocessing stage. Further work is in progress to carry such an implementation is the general case of a parametrized loading history.

On a final note, we observe that all those simplifying features ultimately stem from a distinctive property of SMA models, namely the constraints that are placed on the internal variable. Compared to plasticity, such constraints makes solving incremental evolutions more complicated but they considerably simplify the shakedown analysis.

\section{References}

[1] E. Melan, Sitz.Berl.Ak.Wiss. 145, 195 (1936) 
[2] W.T. Koiter, General problems for elastic solids, Progress in solid mechanics (1960)

[3] A. Constantinescu, K. Dang Van, M. Maitournam, Eng. Mater. Struct. 26, 561 (2003)

[4] S. Govindjee, C. Miehe, Comput. Meth. Appl. Mech. Engrg. 191, 215 (2001)

[5] M. Peigney, C.R.Mecanique 334, 266 (2006)

[6] M. Peigney, J. Seguin, E. Hervé-Luanco, Int. J. Sol. Struct. 48, 2791 (2011)

[7] M. Peigney, J. Seguin, Int. J. Sol. Struct. 50, 4043 (2013)

[8] M. Peigney, Eur. J. Mech. A 29, 785 (2010)

[9] M. Peigney, Ann. Solid Struct. Mech. 6, 17 (2014)

[10] B. Halphen, Q.S. Nguyen, J.Mécanique 14, 1 (1975)

[11] H. Brézis, Opérateurs maximaux monotones et semigroupes de contractions dans les espaces de hilbert, North-Holland, Amsterdam (1972)

[12] M. Frémond, Non-smooth thermomechanics, Springer (2002)

[13] A. Souza, E. Mamiya, N. Zouain, Eur.J.Mech. A 17, 789 (1998)
[14] F. Auricchio, L. Petrini, Int.J.Num.Meth.Eng. 61, 807 (2004)

[15] Q.S. Nguyen, J.Mech.Phys.Solids 51, 101 (2003)

[16] J. Mandel, B. Halphen, J. Zarka, Mech. Res. Comm. 4, 309 (1977)

[17] K. Hackl, R. Heinen, J.Mech.Phys.Solids 56, 2832 (2008)

[18] M. Peigney, J.Mech.Phys.Solids 57, 970 (2009)

[19] M. Peigney, J.Mech.Phys.Solids 61, 1489 (2013)

[20] M. Peigney, J.Mech.Phys.Solids 61, 1511 (2013)

[21] Y.J. Ahn, E. Bertocchi, J. Barber, J.Mech.Phys.Solids 56, 3433 (2008)

[22] M. Peigney, J.Mech.Phys.Solids 71, 112 (2014)

[23] S.H. Duda, M. Bosiers, J. Lammer, D. Scheinert, T. Zeller, A. Tielbeek, J. Anderson, B. Wiesinger, G. Tepe, A. Lansky et al., J. Vasc. Interv. Radiol. 16, 331 (2005)

[24] S. Sabeti, W. Mlekusch, J. Amighi, E. Minar, M. Schillinger, J. Endovasc. Ther. 12, 6 (2005)

[25] A. Pelton, V. Schroeder, M. Mitchell, X.Y. Gong, M. Barney, S. Robertson, J. Mech. Behavior Biomed. Mater. 1, 153 (2008) 